Supporting Information for

\title{
Biomineralization-Inspired Synthesis of Copper
}

\section{Sulfide-Ferritin Nanocages as Cancer Theranostics}

\author{
Zhantong Wang, ${ }^{\dagger}$ Peng Huang, ${ }^{*}{ }^{\star}$ Orit Jacobson, ${ }^{\perp}$ Zhe Wang,,${ }^{\perp}$ Yijing Liu,${ }^{\perp}$ Lisen Lin,$\perp$ \\ Jing Lin, ${ }^{\ddagger}$ Nan Lu, ${ }^{\perp}$ Huimin Zhang, ${ }^{\perp}$ Rui Tian, ${ }^{\dagger},{ }^{\perp}$ Gang Niu, ${ }^{\perp}$ Gang Liu,,${ }^{*} \dagger$ and Xiaoyuan \\ Chen*,1 \\ ${ }^{\dagger}$ State Key Laboratory of Molecular Vaccinology and Molecular Diagnostics \& Center for Molecular Imaging and \\ Translational Medicine, School of Public Health, Xiamen University, Xiamen 361102, China \\ ${ }^{\ddagger}$ Guangdong Key Laboratory for Biomedical Measurements and Ultrasound Imaging, Department of Biomedical \\ Engineering, School of Medicine, Shenzhen University, Shenzhen 518060, China \\ ${ }^{\perp}$ Laboratory of Molecular Imaging and Nanomedicine, National Institute of Biomedical Imaging and Bioengineering, \\ National Institutes of Health, Bethesda, Maryland 20892, United States
}

Corresponding authors: P. Huang (peng.huang@szu.edu.cn); G. Liu (gangliu.cmitm@xmu.edu.cn); X. Chen (hawn.chen@nih.gov) 


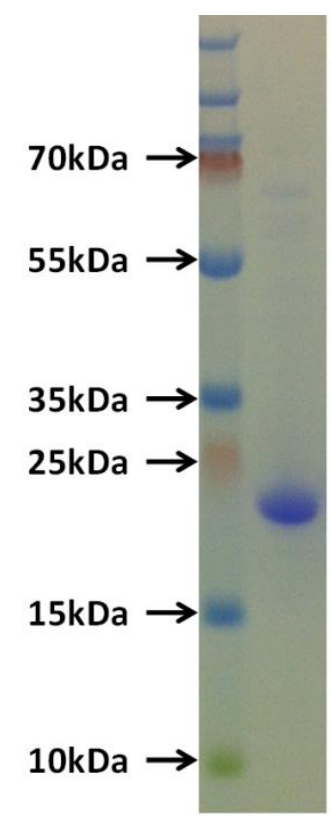

Figure S1. Coomassie blue stained SDS-PAGE image of ferritin and pre-stained protein marker.

$\mathbf{a}$

\section{Translator}

Translate DNA and RNA sequences to protein sequences.

$>$ Home > Translator

Foward frame 1

552 nucleotides, 184 amino acids, structure: sequence

1 ATGGGCACCACCGCG TCTCCCTCGCAAGTG CGCCAGAACTACCAC CAGGACTCGGAGGCT GCCATCAACCGCCAG

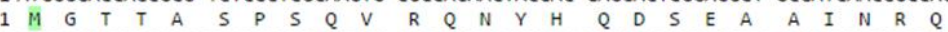
76 ATCAACCTGGAGTTG TATGCCTCCTACGTC TATCTGTCCATGTCT TGTTATTTTGACCGG GATGATGTGGCCCTG

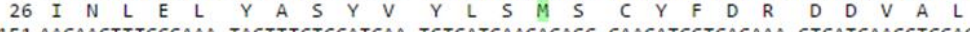
151 AAGAACTTTGCCAAA TACTTTCTCCATCAA TCTCATGAAGAGAGG GAACATGCTGAGAAA CTGATGAAGCTGCAG $\begin{array}{llllllllllllllllllllllllll}51 & K & N & F & A & K & Y & F & L & H & Q & S & H & E & E & R & E & H & A & E & K & L & M & K & L & Q\end{array}$ 226 AACCAGCGAGGTGGA CGAATCTTCCTGCAG GATATAAAGAAACCT GACCGTGATGACTGG GAGAGCGGGCTGAAT $76 \mathrm{~N} O \mathrm{R}$ G G R I F L Q D I K K P D R D D W E S G L N 301 GCAATGGAGTGTGCA CTGCACTTGGAAAAG AGTGTGAATCAGTCA CTACTGGAACTTCAC AAACTGGCTACTGAC 101 A M E C A L H L E K S V N Q S L L E L 376 AAGAATGATCCCCAC TTATGTGACTTCATT GAGACGCATTACCTG AATGAGCAGGTGAAA TCCATTAAAGAACTG

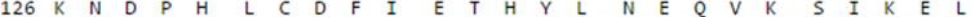
451 GGTGACCACGTGACC AACTTACGCAAGATG GGAGCCCCTGAATCT GGCATGGCAGAATAT CTCTTTGACAAGCAC 151 G $D$ D

b 526 ACCCTGGGACACGGT GATGAGAGCTAA

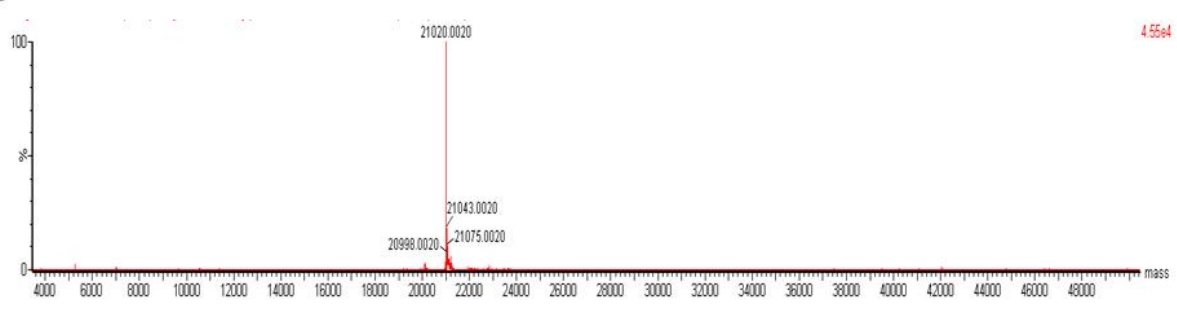

Figure S2. (a) Open reading frame of the rat ferritin heavy chain for CuS-Fn NCs synthesis. (b) ESI-MS analysis of Ferritin. 


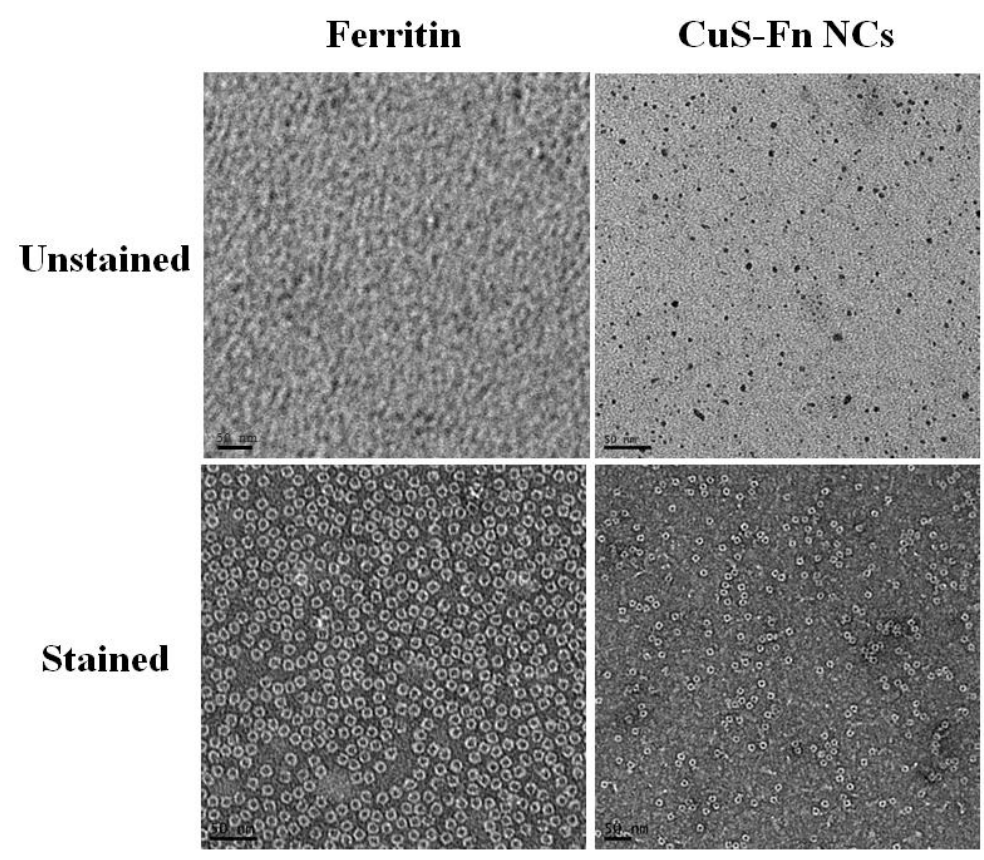

Figure S3. Comparison of ferritin and CuS-Fn NCs samples with or without uranyl acetate staining.
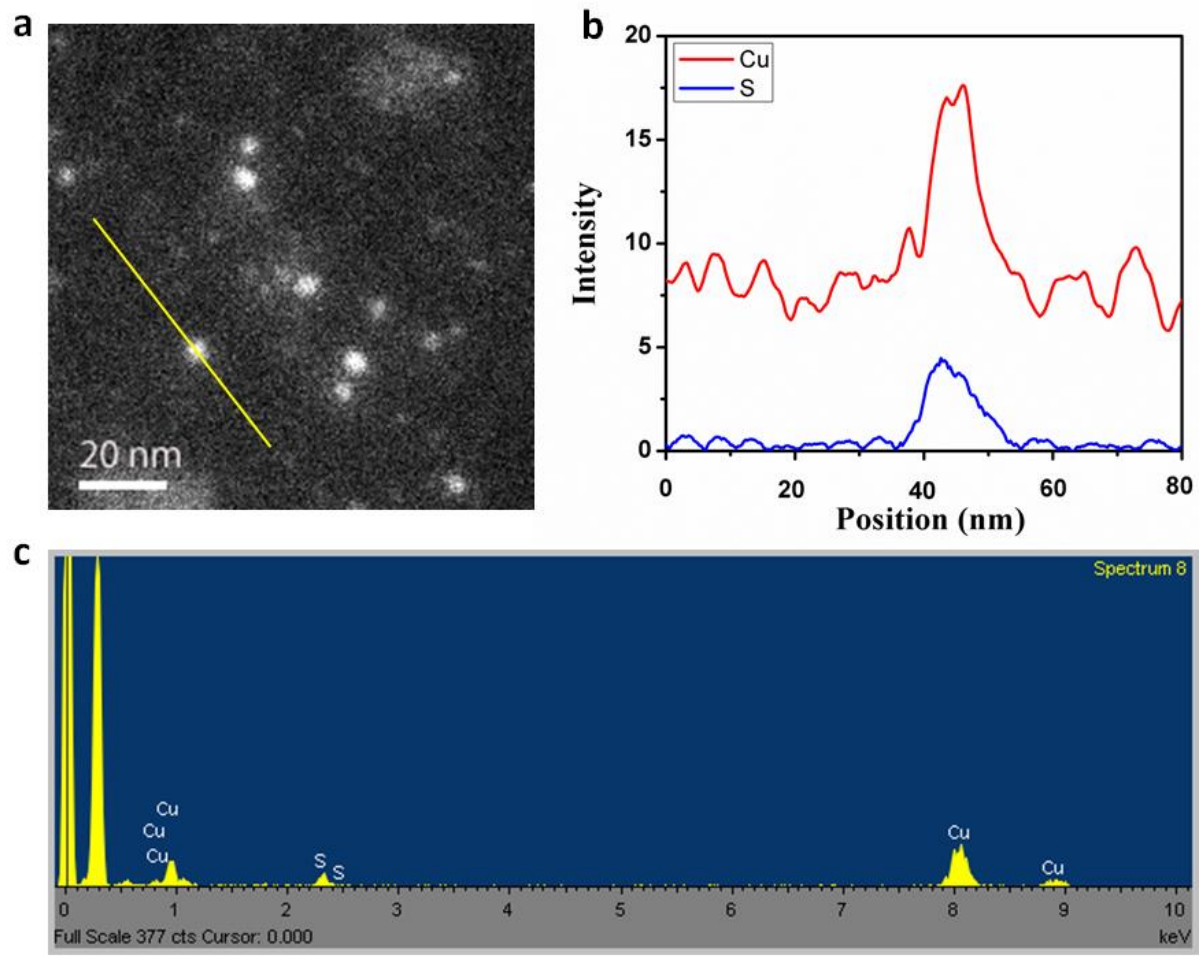

Figure S4. (a) HAADF-STEM image of CuS-Fn NCs. (b) High resolution line-scan EDS analysis of CuS-Fn-NCs. (c) Area-scan EDS analysis of CuS-Fn-NCs. 

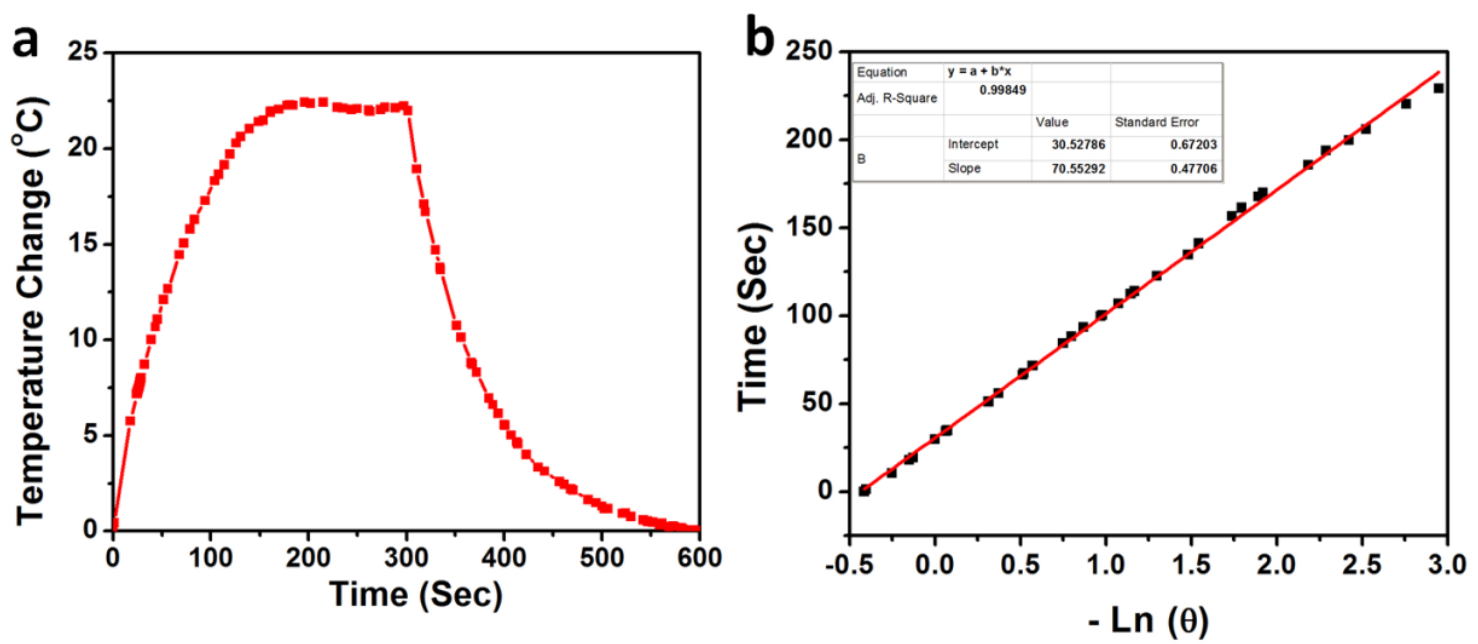

Figure S5. (a) Photothermal effect of the irradiation of the aqueous dispersion of CuS-Fn NCs $(80 \mu \mathrm{g} / \mathrm{mL})$ with the NIR laser $\left(808 \mathrm{~nm}, 0.2 \mathrm{~W} / \mathrm{cm}^{2}\right)$, in which the irradiation lasted for $5 \mathrm{~min}$, and then the laser was turned off. (b) Time constant for heat transfer from the system is determined to be $\tau_{\mathrm{s}}=70.55 \mathrm{~s}$ by applying the linear time data from the cooling period (after 300 s) versus negative natural logarithm of driving force temperature, which is obtained from the cooling stage of panel 1 .
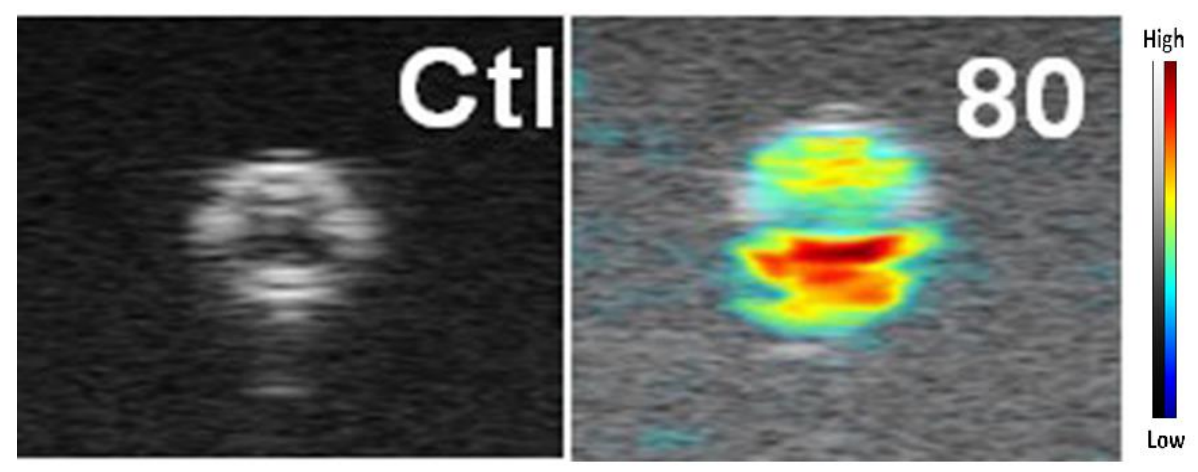

Figure S6. Photoacoustic images of PBS (Control) and CuS-Fn $(80 \mu \mathrm{g} / \mathrm{mL})$ solution. 


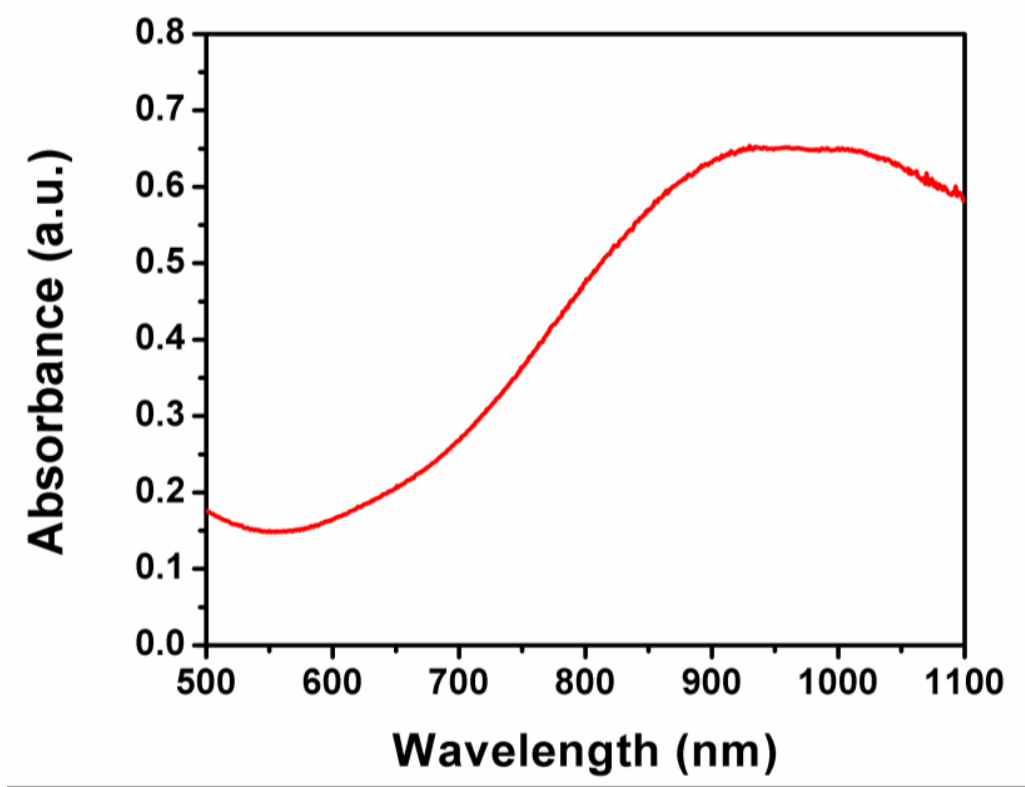

Figure S7. UV-vis absorbance spectrum of CuS nanoparticle.
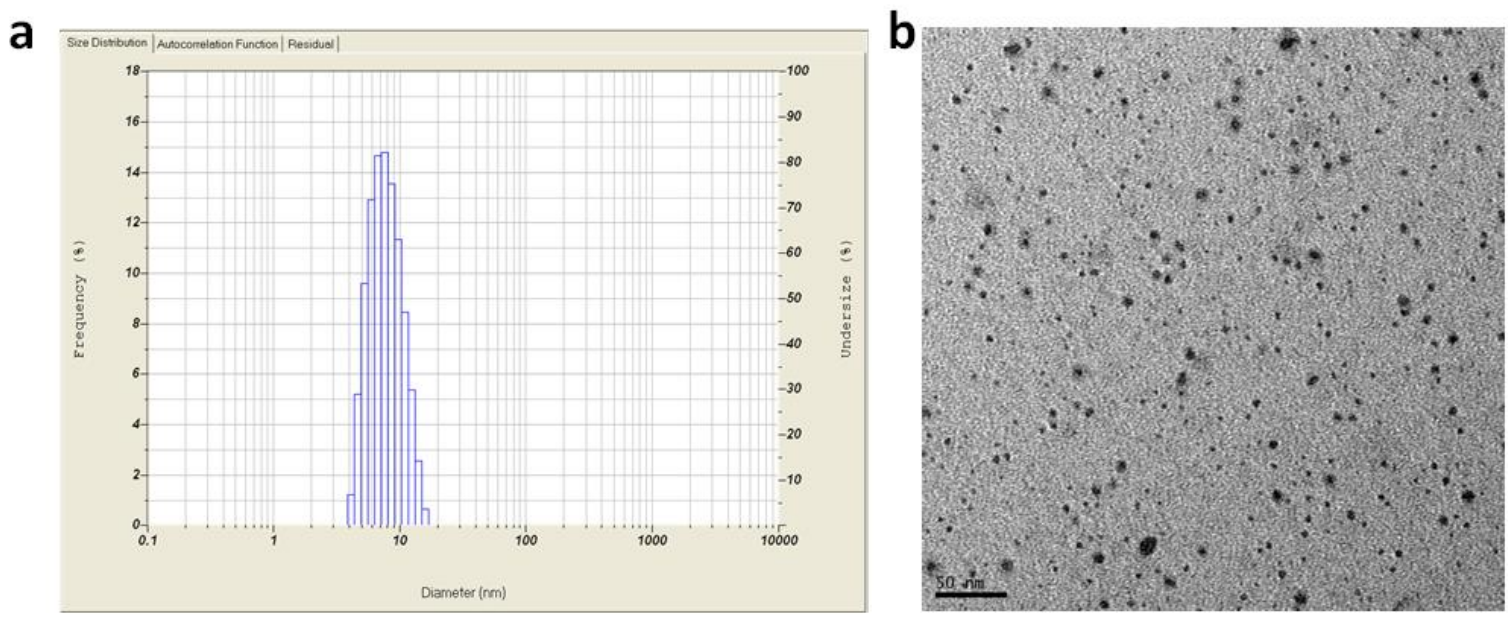

Figure S8. (1) DLS analysis of CuS nanoparticle. (2) TEM image of CuS nanoparticle . 
a

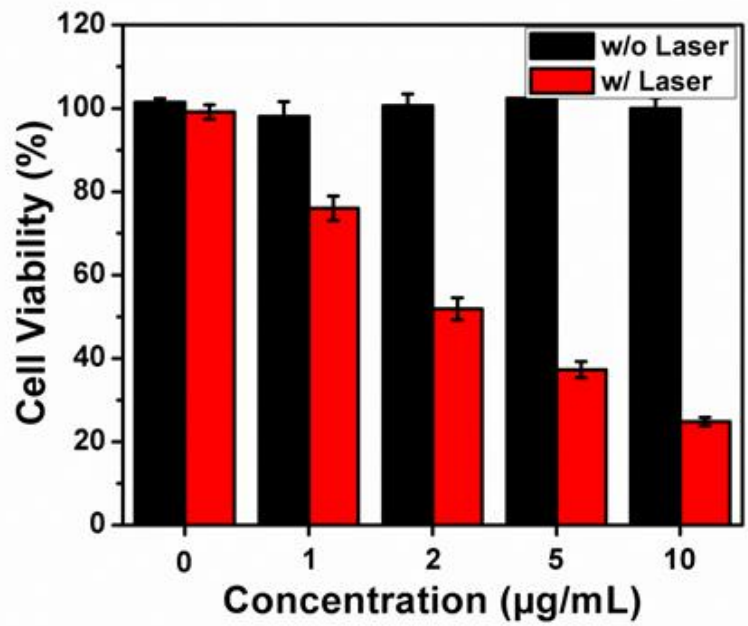

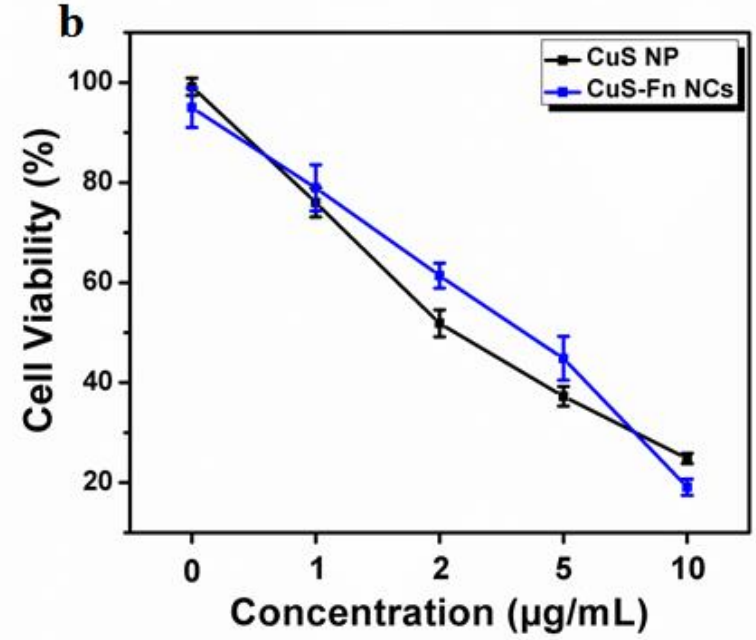

Figure S9. (a) Cell toxicity and photothermal efficiency of CuS. (b) Photothermal efficiency comparison between $\mathrm{CuS}$ nanoparticle and CuS-Fn NCs. 


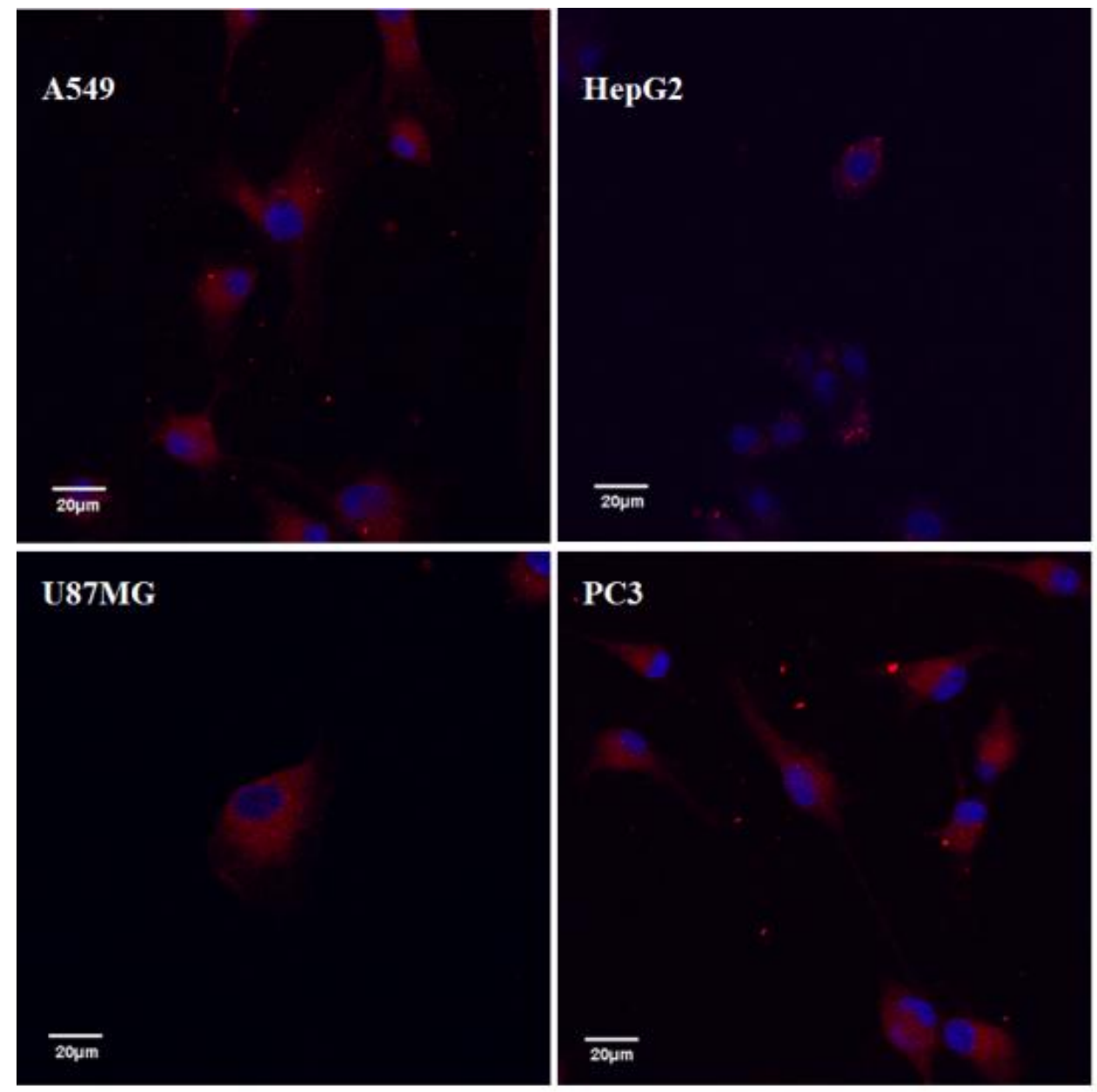

Figure S10. Cellular uptake of CuS-Fn-Cy 5.5 on different cell lines: U87MG, A549, PC3, HepG2 cells. 


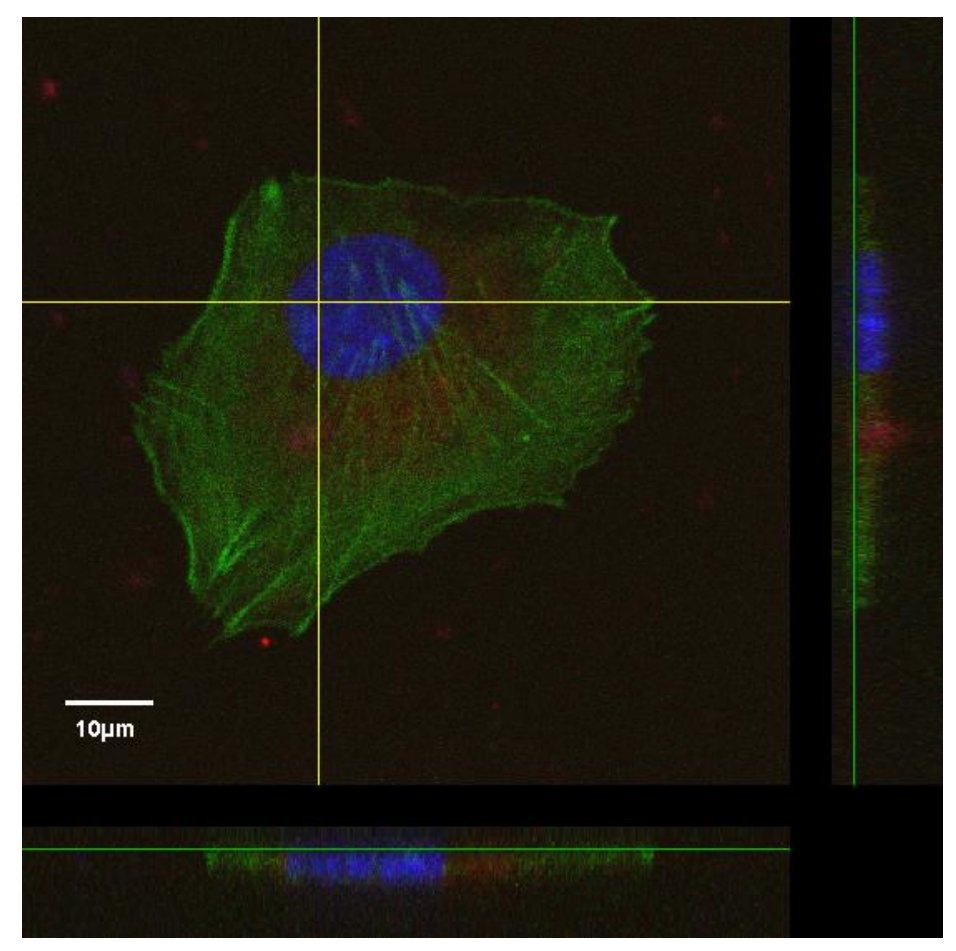

Figure S11. Multi-slice confocal image of U87 MG cell after CuS-Fn-Cy 5.5 treatment. (Blue: DAPI, Green: Alexa 488 conjugated phalloidin, Red: CuS-Fn-Cy 5.5)
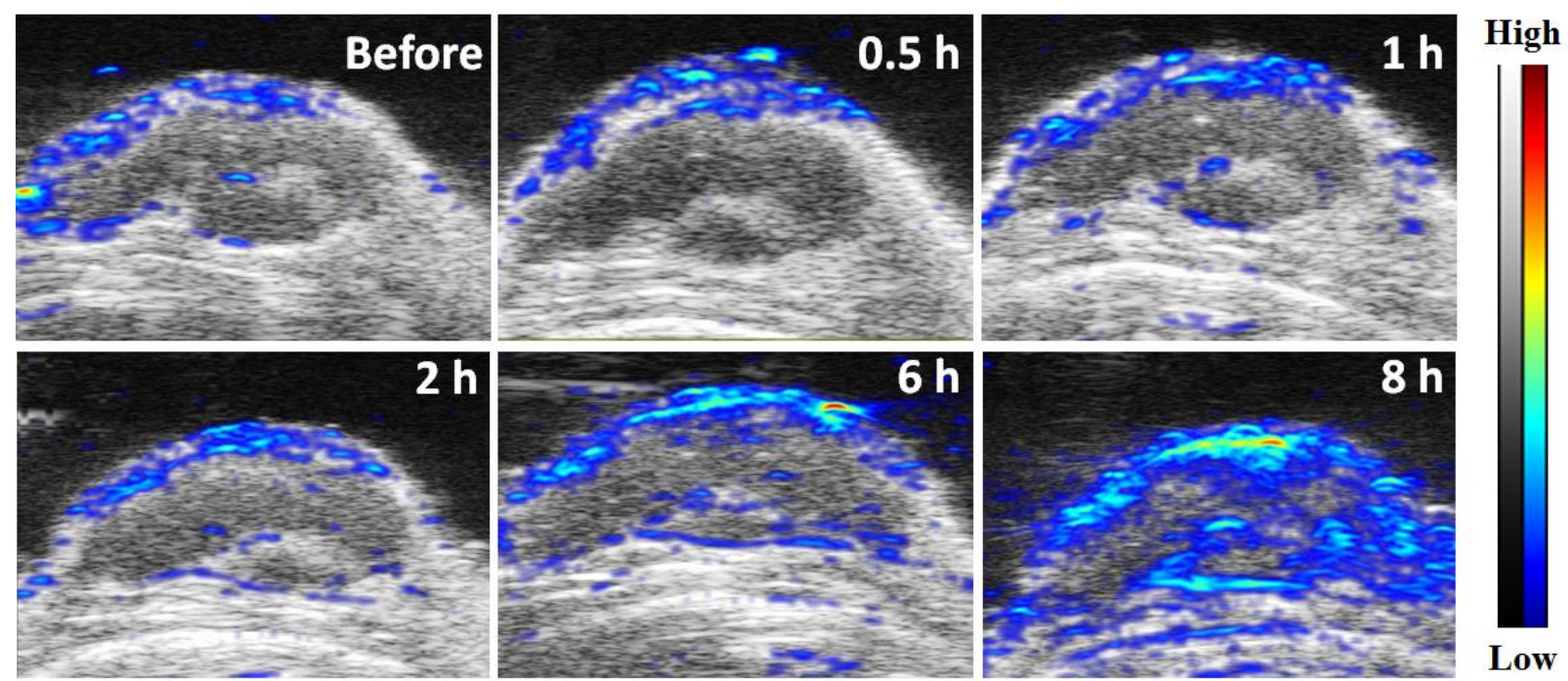

Low

Figure S12. 2D photoacoustic images of U87 MG tumor of different time point after CuS-Fn NCs injection. 

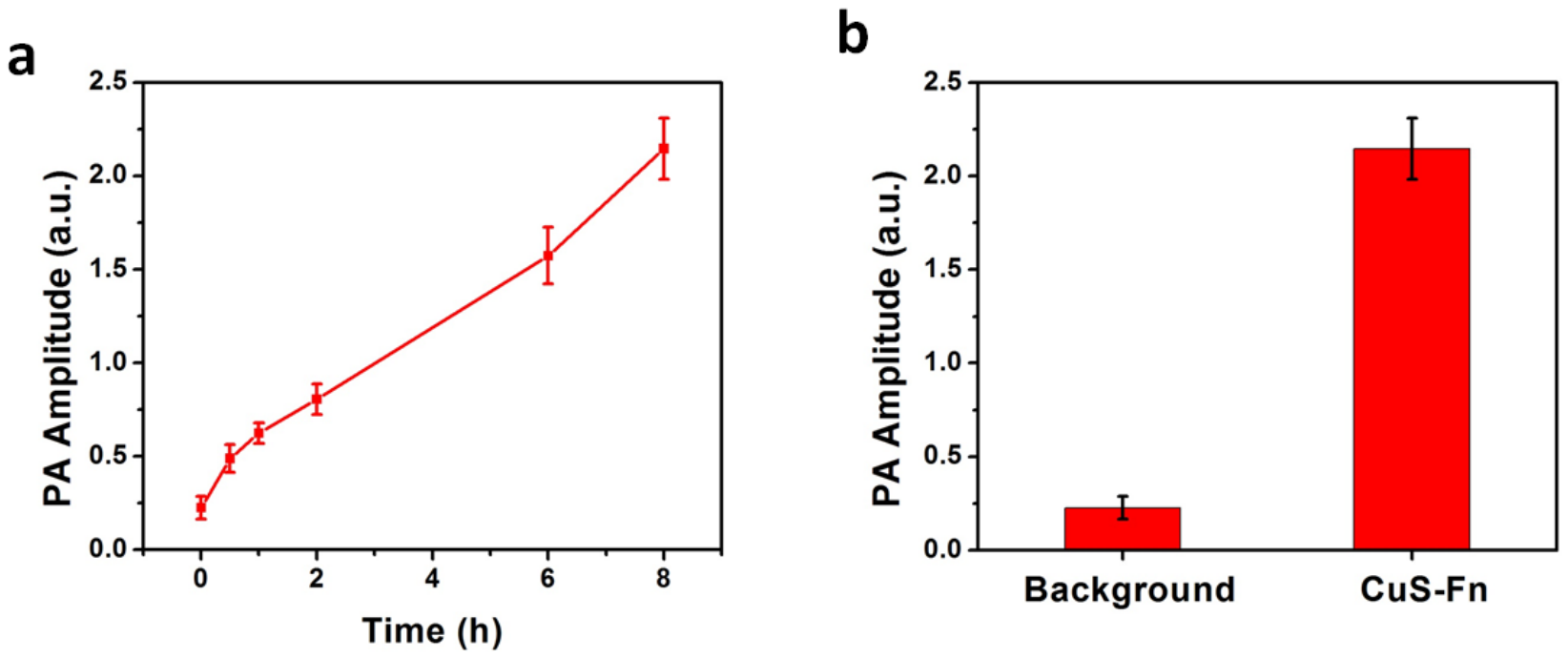

Figure S13. a. PA signal of tumor region amplitude after CuS-Fn NCs injection. b. PA amplitude comparison between tumor region and background.
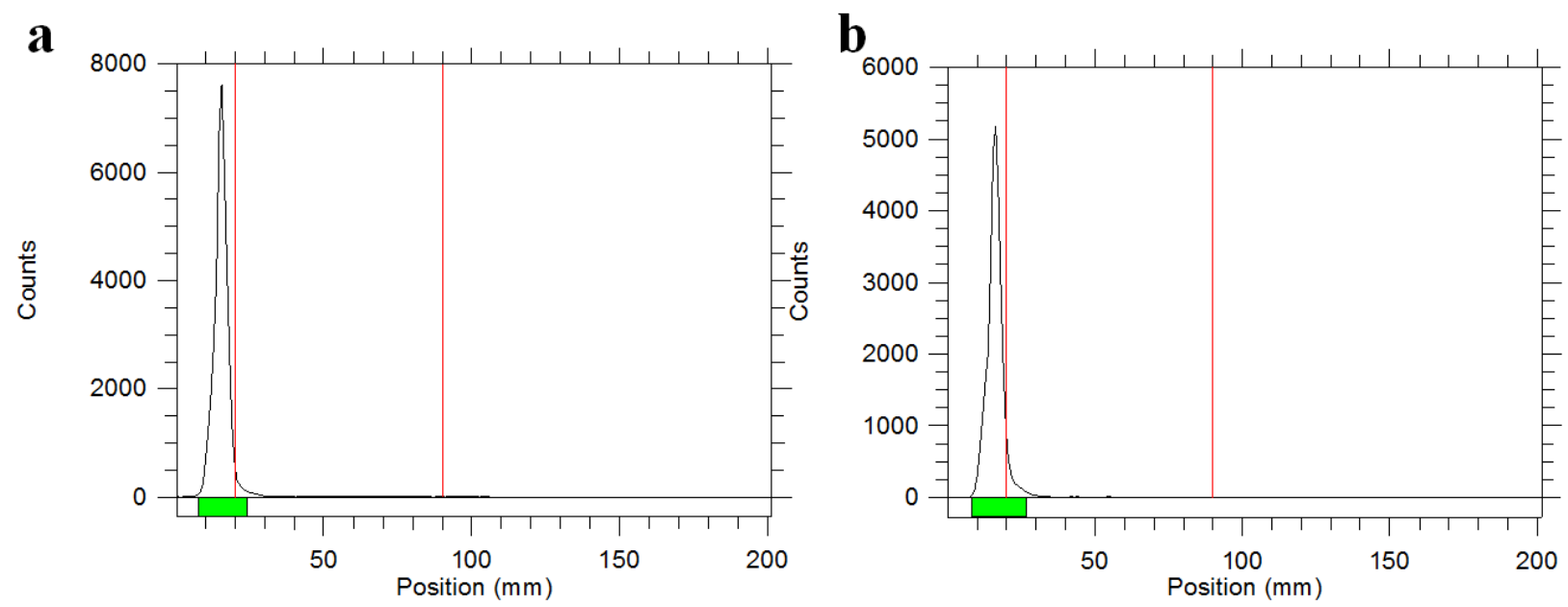

Figure S14. TLC plate analyses of ${ }^{64} \mathrm{CuS}-\mathrm{Fn}$ NCs after incubation with Na-acetate for 10 min (a) and $24 \mathrm{~h}(\mathrm{~b})$. 


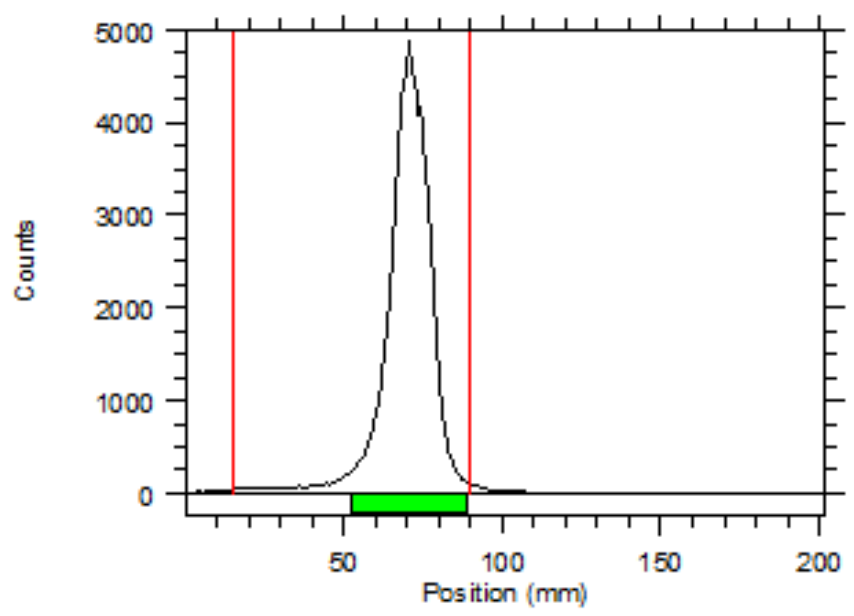

Figure S15. TLC plate analyses of ${ }^{64} \mathrm{Cu}$-acetate.

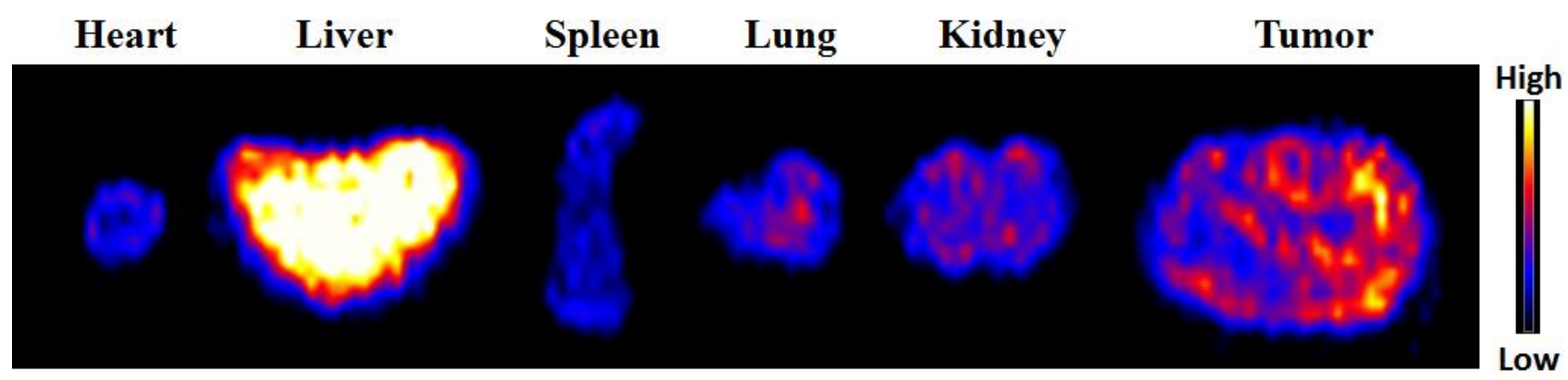

Figure S16. Ex vivo PET image of major organ and tumor $24 \mathrm{~h}$ after CuS-Fn NCs injection.

\section{Control}

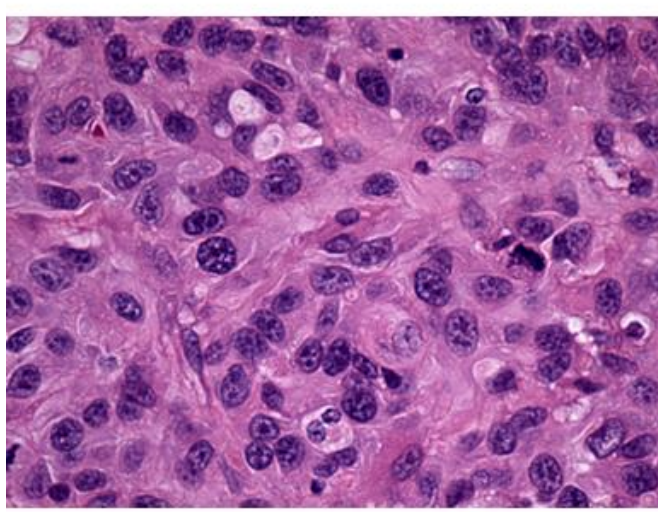

Particle w/ Laser

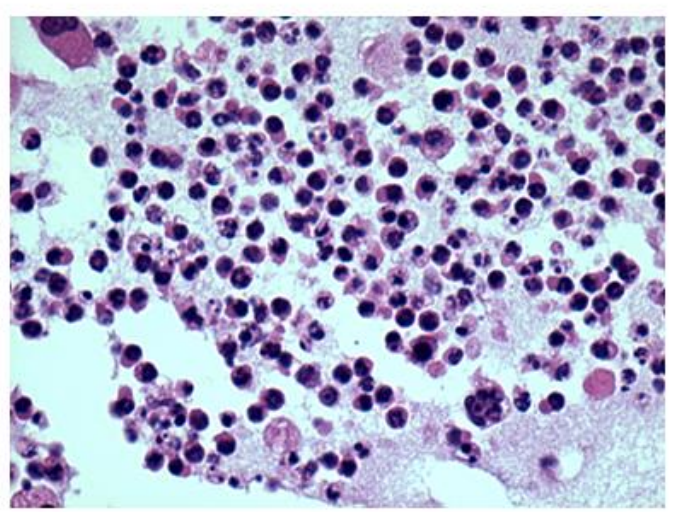

Figure S17. H\&E staining of tumors. 


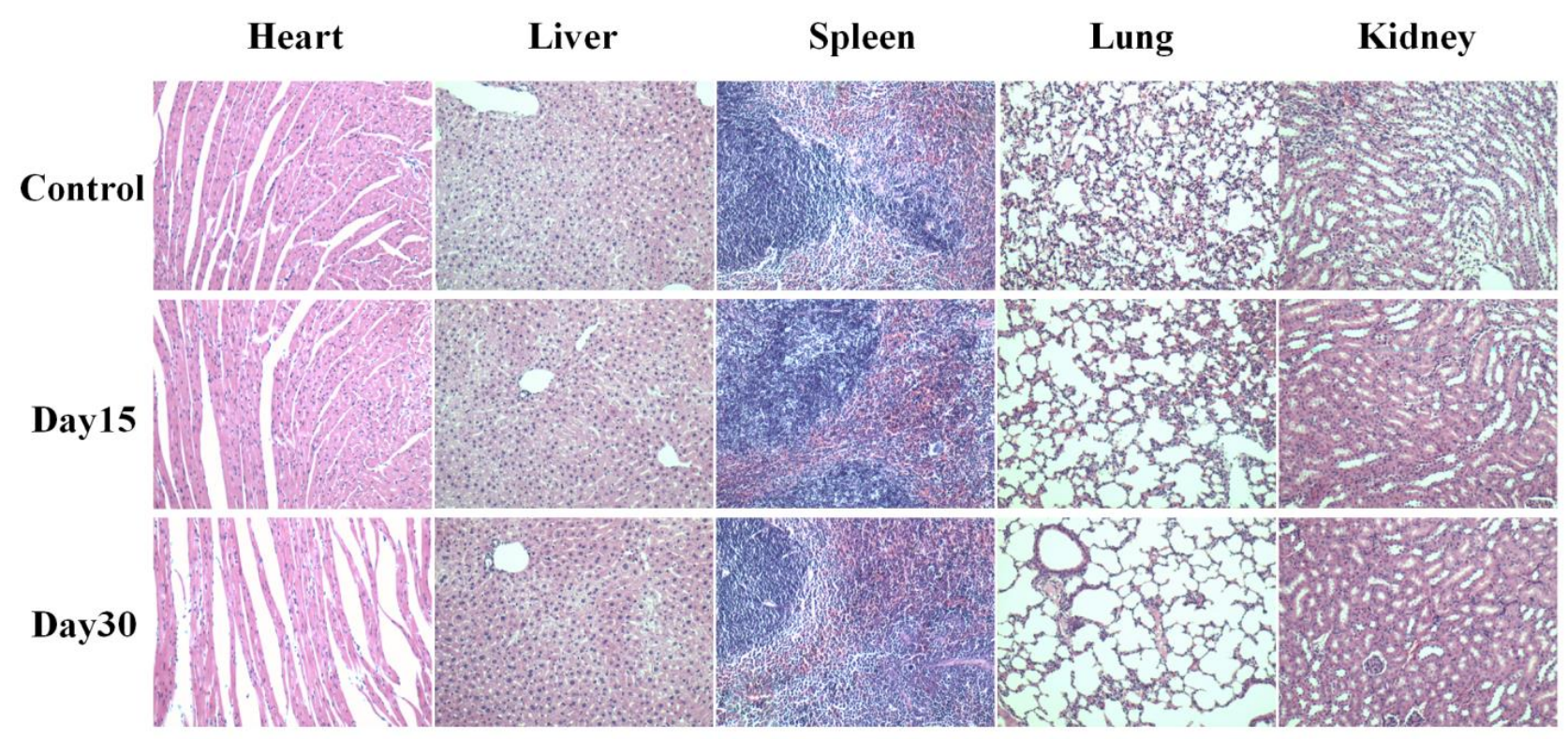

Figure S18. H\&E staining of major organs. 